\title{
Osteochondroma of the Distal Clavicle: A Rare Cause of Impingement and Biceps Tear of the Shoulder
}

\author{
Dong-Wan Kim, Ki-Cheor Bae, Eun-Seok Son, Chung-Sin Baek, Chul-Hyun Cho \\ Department of Orthopedic Surgery, Dongsan Medical Center, Keimyung University School of Medicine, Daegu, Korea
}

\begin{abstract}
Hereditary multiple exostosis (HME) is an autosomal dominant disorder manifested by the presence of multiple osteochondromas. Although the lesions are benign in nature, exostoses are often associated with characteristic progressive skeletal deformity and displaying clinical symptoms such as mechanical irritation or impingement. We present the successful arthroscopic resection in a 24-year-old HME male with impingement syndrome and long head tendon tear of the biceps caused by osteochondroma arising from the distal clavicle.
\end{abstract}

(Clin Shoulder Elbow 2018;21(3):158-161)

Key Words: Clavicle; Hereditary multiple exostoses; Shoulder impingement syndrome; Biceps tendon

Hereditary multiple exostosis (HME) is an autosomal dominant disorder manifested by the presence of multiple osteochondromas. ${ }^{1)}$ Although exostoses are benign lesions, they are often associated with characteristic progressive skeletal deformity and may cause clinical symptoms such as mechanical irritation or impingement. ${ }^{1)}$ Due to the shoulder anatomy, direct contact of the protruding osteochondroma originating at the scapula, clavicle, or proximal humerus can result in mechanical impingement, ${ }^{2-7)}$ and could be a possible cause of tendon tear. We present here the successful arthroscopic resection in a 24-year-old HME male who presented with impingement syndrome and long head tendon tear of the biceps caused by osteochondroma arising from the distal clavicle.

\section{Case Report}

A 24-year-old right-handed male college student presented with right shoulder pain and limited motion for 6 months, with no history of trauma to the shoulder. He had been diagnosed and treated as HME from childhood, and had undergone corrective tibia osteotomy for both genu valgum 12 years ago. The passive range of motion of right shoulder was 90 degrees of forward flexion, 100 degrees of abduction, 10 degrees of external rotation at the side, and L1 level internal rotation towards the back. Impingement signs, Speed test, and Yergason test were positive. The neurovascular examination was normal.

Multiple osteochondromas of the distal clavicle, scapula, and proximal humerus were observed in the plain radiographs of the right shoulder. Three-dimensional computed tomography scan revealed a $23 \times 15 \times 28 \mathrm{~mm}$-sized pedunculated mass originating from the distal clavicle and protruding to the anterosuperior area of the shoulder joint (Fig. 1). Magnetic resonance imaging scans showed that osteochondroma of the distal clavicle was compressing the long head of the biceps tendon through the rotator interval (Fig. 2).

Based on the above findings and a presumptive diagnosis of shoulder impingement syndrome by osteochondroma of the distal clavicle, an arthroscopic mass resection was planned. Examination under general anesthesia showed that the passive range of motion was same as perceived during preoperative examination. The patient was placed in the lateral decubitus position, and a standard arthroscopic glenohumeral examination was

Received May 20, 2018. Revised July 8, 2018. Accepted July 10, 2018.

Correspondence to: Chul-Hyun Cho

Department of Orthopedic Surgery, Dongsan Medical Center, Keimyung University School of Medicine, 56 Dalseong-ro, Jung-gu, Daegu 41931, Korea

Tel: +82-53-250-8160, Fax: +82-53-250-7205, E-mail: oscho5362@dsmc.or.kr, ORCID: https://orcid.org/0000-0003-0252-8741

IRB approval: Keimyung University Dongsan Medical Center (No. DSMC 2018-05-041).

Financial support: None. Conflict of interests: None. 


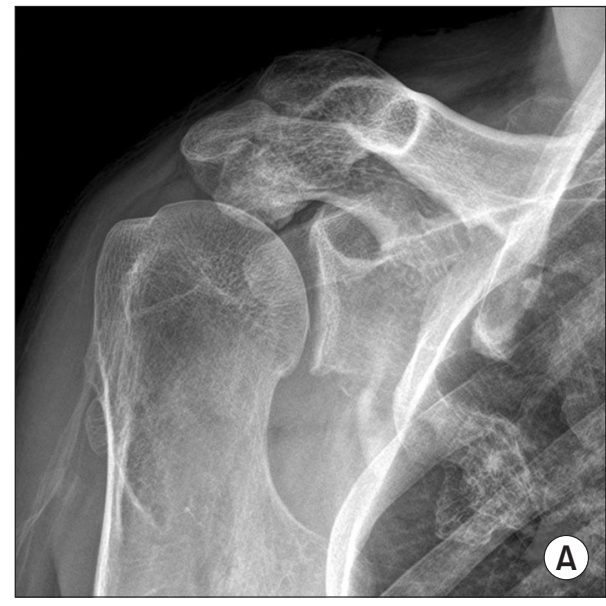

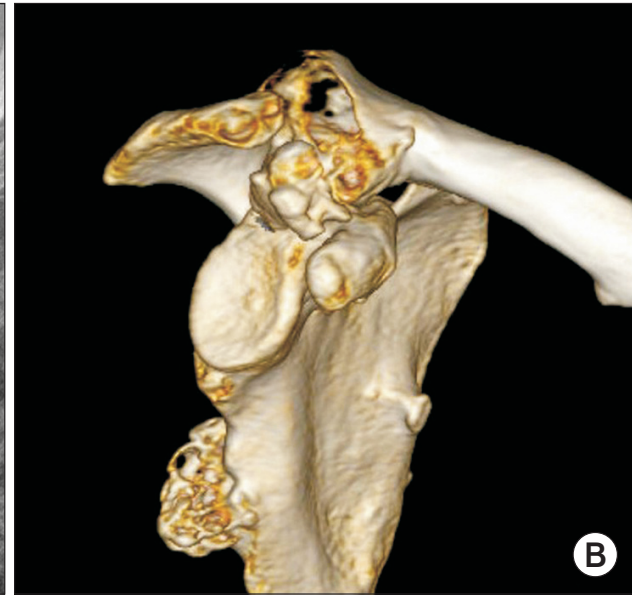

B

Fig. 1. Anteroposterior plain radiograph (A) and computed tomography scan (B) show osteochondromas of the distal clavicle, scapula, and proximal humerus.
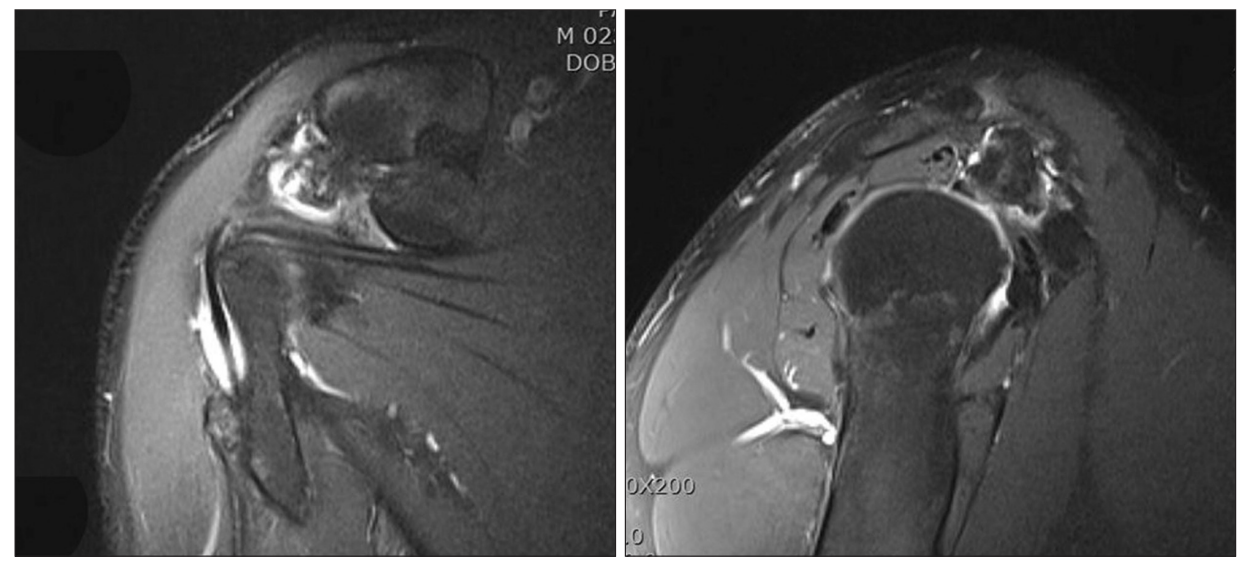

Fig. 2. Magnetic resonance imaging scans show that osteochondroma of the distal clavicle compressed long head of the biceps tendon through the rotator interval. performed through the posterior and anterior portals to evaluate lesions of the shoulder joint. We found the pedunculated osteochondroma had protruded into the rotator interval, and the long head of the biceps tendon was partially torn due to attrition of the mass. Arthroscopic debridement was performed for partial tear of long head of the biceps tendon. Briefly, the arthroscope was placed in the subacromial space. Lateral and posterolateral portals were created as working and viewing portal for mass resection, following which the mass was resected sufficiently using an acromionizer burr (Fig. 3).

Passive range of motion exercise were initiated immediately after surgery. Pain and range of motion of the shoulder dramatically improved at 6 weeks after surgery. The passive range of motion was 160 degrees of forward flexion, 150 degrees of abduction, 40 degrees of external rotation at the side, and T6 level internal rotation behind. At 2 years after surgery, there has been no recurrence and the patient remains asymptomatic (Fig. 4).

\section{Discussion}

Mechanical irritation of the subacromial bursa and rotator cuff by the coracoacromial arch remains the most common cause of impingement syndrome. Offending structures include the undersurface of the anterolateral acromion, coracoacromial ligament, and the undersurface of the distal clavicle.

Osteochondroma around shoulder joint including the acromion, proximal humerus or clavicle rarely cause impingement syndrome or tendon rupture. ${ }^{2-7)}$ To date, three studies report on 4 cases in which extrinsic impingement resulted from a distal clavicle osteochondroma. ${ }^{3,4,7)}$ Craig $^{3)}$ reported a 32-year-old HME female with multiple osteochondromas arising from the distal clavicle and undersurface of the acromion that resulted in mechanical impingement. The patient was treated with open excision of the osteochondroma, acromioplasty, and distal clavicle excision. Fallon and Hollinshead ${ }^{4)}$ reported a 28-year-old female with solitary osteochondroma of the distal clavicle causing a fullthickness rotator cuff tear. The patient was treated with open mass resection, distal clavicle excision, and rotator cuff repair. Reichmister et al. ${ }^{7)}$ reported 2 cases with osteochondroma of the distal clavicle. The first patient (a 33-year-old male) underwent open surgery with decompression of the coracoacromial arch, distal clavicle excision (including osteochondroma), and repair of a nearly full-thickness bursal rotator cuff tear. The other patient (a 32-year-old female) underwent an open distal clavicle exci- 

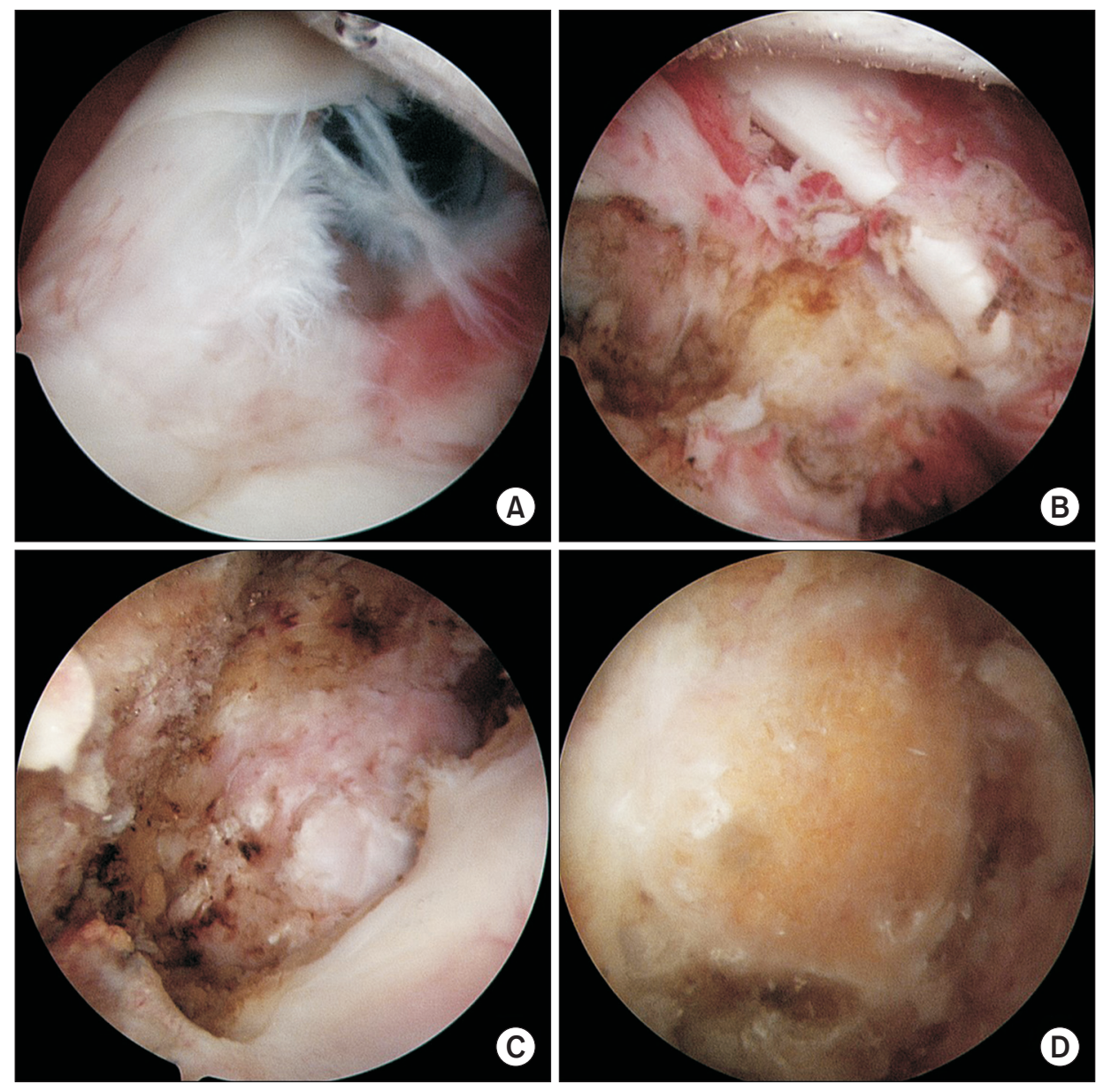

Fig. 3. Arthroscopic images: (A) The pedunculated osteochondroma protrude into rotator interval. (B) Attritional tear of the long head of biceps tendon by bony mass. (C) Subacromial view. (D) After resection.
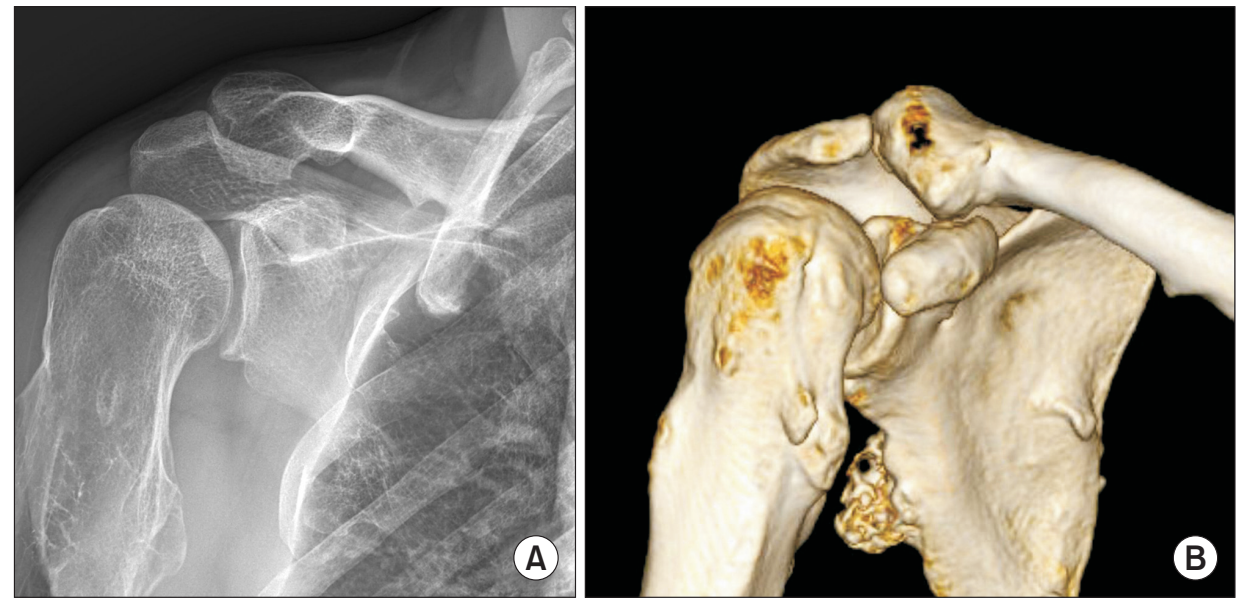

Fig. 4. Anteroposterior plain radiograph (A) and computed tomography scan (B) taken at 2 years after surgery show no recurrence.

sion involving only the osteochondroma. In the current study, a 24-year-old male with HME presented painful limited motion of the shoulder joint. Consolidating his history and all clinical assessments, we confirmed an osteochondroma arising from the distal clavicle growing toward the rotator interval, resulting in impingement syndrome and attrition of the biceps long head tendon tear.
To offer the best opportunity and prevention of recurrence, previous studies recommend open distal clavicle excision with tumor removal rather than extraperiosteal resection. ${ }^{3,4,7)}$ However, in our case, we achieved complete excision of the osteochondroma arising from the distal clavicle using an arthroscopic technique. The patient remains symptom-free without any recurrence at 2 years after surgery. We believe that with the 
development of new arthroscopic instruments and techniques, arthroscopic surgery is capable of producing similar or better outcomes than open surgery. According to the localization, mass size, and experience of a surgeon, an appropriate procedure (including open or arthroscopic technique) can be used to excise the mass.

To the best of our knowledge, this is the first report of arthroscopic resection of an osteochondroma arising distal clavicle. We propose that osteochondroma of the distal clavicle needs to be encompassed as a cause of painful limitation of shoulder rotation or tear of the biceps tendon. Arthroscopic resection of the osteochondroma and reactive synovitis is an effective method for eliminating the mechanical impingement syndrome, thereby recovering function and improving pain relief.

\section{References}

1. Stieber JR, Dormans JP. Manifestations of hereditary multiple exostoses. J Am Acad Orthop Surg. 2005;13(2):110-20.

2. Çıtlak A, Akgün U, Bulut T, Aslan C, Mete BD, Şener M. Subacromial osteochondroma: a rare cause of impingement syndrome. Int J Surg Case Rep. 2015;6:126-8.

3. Craig EV. Subacromial impingement syndrome in hereditary multiple exostoses. Clin Orthop Relat Res. 1986;(209):182-4.

4. Fallon PJ, Hollinshead RM. Solitary osteochondroma of the distal clavicle causing a full-thickness rotator cuff tear. J Shoulder Elbow Surg. 1994;3(4):266-9.

5. Kim JR, Lee JH, Wang SI. Arthroscopic management of lesser tuberosity osteochondroma causing impingement and subscapularis tear of the shoulder. Knee Surg Sports Traumatol Arthrosc. 2017;25(7):2225-9.

6. Lu MT, Abboud JA. Subacromial osteochondroma. Orthopedics. 2011;34(9):e581-3.

7. Reichmister J, Reeder JD, Gold DL. Osteochondroma of the distal clavicle: an unusual cause of rotator cuff impingement. Am J Orthop (Belle Mead NJ). 2000;29(10):807-9. 